\title{
Menstrual Cycle and the Temporal Discrimination Threshold
}

\author{
Eavan M. Mc Govern \\ Department of Neurology, St. Vincent's University Hospital, Dublin, Ireland \\ Emer O'Connor \\ Department of Neurology, St. Vincent's University Hospital, Dublin, Ireland \\ Ines Beiser \\ School of Medicine and Medical Sciences, University College Dublin, Dublin, Ireland
}

See next page for additional authors

Follow this and additional works at: https://arrow.tudublin.ie/scschmatart

Part of the Mathematics Commons, and the Medicine and Health Sciences Commons

\author{
Recommended Citation \\ McGovern, E.M., O'Connor, E. \& Beiser, I. (2018). Menstrual Cycle and the Temporal Discrimination \\ Threshold. Physiological Measurement, vol. 38, no. 2, pg. 65-72. doi: 10.1088/1361-6579/38/2
}

This Article is brought to you for free and open access by the School of Mathematics at ARROW@TU Dublin. It has been accepted for inclusion in Articles by an authorized administrator of ARROW@TU Dublin. For more information, please contact arrow.admin@tudublin.ie, aisling.coyne@tudublin.ie,gerard.connolly@tudublin.ie.

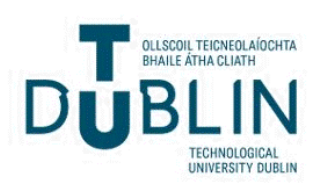




\section{Authors}

Eavan M. Mc Govern, Emer O'Connor, Ines Beiser, Laura Williams, John Butler, Brendan Quinlivan, Shruti Narasimham, Rebecca Beck, Richard B. Reilly, Sean O'Riordan, and Michael Hutchinson 
NOTE

\section{Menstrual cycle and the temporal discrimination threshold}

To cite this article: Eavan M Mc Govern et al 2017 Physiol. Meas. 38 N65

View the article online for updates and enhancements.

\section{Related content}

Non-parametric bootstrapping method for measuring the temporal discrimination threshold for movement disorders John S Butler, Anna Molloy, Laura Williams et al.

- A comparison of stimulus presentation methods in temporal discrimination testing Eavan M Mc Govern, John S Butler, Ines Beiser et al.

- Diary 


\title{
Menstrual cycle and the temporal discrimination threshold
}

\section{Eavan M Mc Govern',2,3, Emer O'Connor ${ }^{1}$, Ines Beiser ${ }^{1,2}$, Laura Williams $^{1,2}$, John S Butler ${ }^{3,4}$, Brendan Quinlivan 3,4 , Shruti Narasimham ${ }^{3}$, Rebecca Beck ${ }^{3}$, Richard B Reilly ${ }^{3,4,5}$, Sean O'Riordan ${ }^{1,2}$ and Michael Hutchinson ${ }^{1,2}$}

${ }^{1}$ Department of Neurology, St. Vincent's University Hospital, Dublin, Ireland 2 School of Medicine and Medical Sciences, University College Dublin, Dublin, Ireland

3 Trinity Centre for Bioengineering, Dublin, Ireland

${ }^{4}$ School of Mathematical Sciences, Dublin Institute of Technology, Dublin, Ireland

5 School of Medicine Trinity College Dublin, The University of Dublin, Dublin, Ireland

E-mail: eavanmcgov@hotmail.com and e.mcgovern@st-vincents.ie

Received 13 July 2016, revised 7 October 2016

Accepted for publication 1 November 2016

Published 18 January 2017

\begin{abstract}
The temporal discrimination threshold (TDT) is a proposed pre-clinical biomarker (endophenotype) for adult onset isolated focal dystonia (AOIFD). Age- and sex-related effects on temporal discrimination demonstrate that women, before the age of 40 years, have faster temporal discrimination than men but their TDTs worsen with age at almost three times the rate of men. Thus after 40 years the TDT in women is progressively worse than in men. AOIFD is an increasingly female-predominant disorder after the age of 40; it is not clear whether this age-related sexually-dimorphic difference observed for both the TDT and sex ratio at disease onset in AOIFD is a hormonal or chromosomal effect. The aim of this study was to examine temporal discrimination at weekly intervals during two consecutive menstrual cycles in 14 healthy female volunteers to determine whether physiological hormonal changes affected temporal discrimination. We observed no significant differences in weekly temporal discrimination threshold values during the menstrual cycles and no significant correlation with the menstrual cycle stage. This observed stability of temporal discrimination during cyclical hormonal change raises interesting questions concerning the age-related sexuallydimorphic decline observed in temporal discrimination. Our findings pave the way for future studies exploring potential pathomechanisms for this agerelated deterioration.
\end{abstract}


Keywords: temporal discrimination, menstrual quartile, oestrogen, sexual dimorphism, adult onset focal dystonia

(Some figures may appear in colour only in the online journal)

\section{Introduction}

The temporal discrimination threshold (TDT) represents the point at which an individual determines two sequential sensory stimuli to be asynchronous (normally about $30-50 \mathrm{~ms}$ ) (Kimmich et al 2011). It has been proposed as a mediational endophenotype for cervical dystonia, the most common form of adult onset idiopathic isolated focal dystonia (AOIFD), a condition with an increased prevalence in women (F:M 2:1) (Hutchinson et al 2013). Mean TDT scores increase with age in a sexually-dimorphic pattern; women have faster temporal discrimination (shorter TDTs) than men between 20 and 40 years of age. After 40 years of age women's TDT scores deteriorate at approximately three times the rate of their male peers (Butler et al 2015, Williams et al 2015). Before this age, women have faster temporal discrimination than men. It is unclear as to whether this age-related sexual dimorphism in temporal discrimination is hormonally or sex chromosome mediated. Menopause is the female transition into reproductive senescence and is accompanied by reduced oestrogen and progesterone levels (Speroff et al 1999). The mean age of menopause occurs at 51.3 years, 10 years after this observed progressive worsening of women's TDT scores relative to men (McKinlay et al 1992). The menstrual cycle is a physiological, monthly occurrence regulated by cyclical-hormonal changes (Mihm et al 2011). Differences in neurophysiological recordings have previously been observed during different phases of the menstrual cycle (Soares et al 2011). This cycle provides an opportunity to examine temporal discrimination during monthly fluctuations in sex-hormone levels. The purpose of this study, therefore, was to examine TDTs weekly during two consecutive menstrual cycles and to correlate TDT scores with individual menstrual cycle stages. We hypothesised that TDT scores would be unaffected by monthly fluctuations in sex-hormone levels. By enhancing our understanding of this mediational endophenotype, we aim to better comprehend the pathomechanisms underlying basalganglia disorders, such as cervical dystonia.

\section{Methods}

\section{Participants}

Fifteen healthy, nulliparous female volunteers were recruited to the study; all were medical students. Exclusion criteria were current or previous use of hormonal contraception and a history of neurological disease. Fourteen participants completed the study. Study participants ranged in age from 19 to 23 years with a mean age of 19 years (SD: \pm 0.99 years). Written informed consent was obtained from all participants. The study was approved by the Medical Research Ethics Committee at St Vincent's University Hospital.

\section{TDT testing}

Visual TDT testing was carried out as described previously (Molloy et al 2014) using a portable-TDT headset, which ensured that participants had a consistent position relative to the stimulus. Two white LED lights were positioned $7^{\circ}$ into the participant's peripheral visual field; 
testing was carried out in a single session in a dimly-lit room. The participant was instructed to fixate on a centrally presented red LED. Initially, the pair of white LEDs flashed synchronously and thereafter illuminated progressively out of sync in $5 \mathrm{~ms}$ steps. The trial ended when the participants reported on three consecutive occasions that the pairs of white LEDs flashed asynchronously. The first of three asynchronous responses was taken as the TDT for that trial. This procedure was repeated four times on the left and right side of the body resulting in a total of eight runs per participant. The order was randomised to minimise practice effects. TDT testing was performed weekly for nine consecutive weeks. The first week of recording was discarded to allow for practice effect.

\section{Menstrual cycle diary}

Participants were asked to keep a menstrual diary. The onset and duration of the menstrual flow and the total-cycle length was noted. The first day of the menstrual flow corresponded to day one of the menstrual cycle. The textbook menstrual cycle is $28 \mathrm{~d}$ (interval between day one of menses to the next menses) (Speroff et al 1999). However, menstrual cycle is highly variable between women and ranges from 24 to $35 \mathrm{~d}$ (Mihm et al 2011). Due to this cyclelength variability, we expressed an individual's menstrual cycle as a quartile of the total-cycle length. Typically, the first $4 \mathrm{~d}$ of menstruation (or the first quartile of a cycle with variablecycle length) corresponds to the early-follicular phase. It is associated with low oestradiol levels, sometimes within post-menopausal levels, low progesterone levels and an elevation in FSH (Sundström Poromaa and Gingnell 2014). This phase was selected as our quartile of interest given its hormonal similarities to menopause (Ycaza Herrera and Mather 2015). Participants were instructed to keep a menstrual diary throughout the testing period and one month either side of this time. Participants entered the study on the same date, thus their testing commenced at varying stages of their menstrual cycle and at different menstrual quartiles.

\section{Statistical analysis}

\section{Temporal discrimination threshold}

The median of four trials on each side was averaged to obtain a summary visual TDT score. All TDT results (in ms) were converted to standardised $Z$-scores to enable comparison of individual results using the formula:

$$
Z \text {-Score }=\frac{\text { Actual TDT }- \text { Age-related control mean TDT }}{\text { Age-related control standard deviation }}
$$

The control mean and standard deviation used in the formula depends on the age of the participant; the age-related control mean TDT (and standard deviation) values have been previously published (Molloy et al 2014).

\section{Participant data psychometric fit}

A cumulative Gaussian psychometric function was fitted to the asynchronous responses as described previously (Butler et al 2015). We extracted the TDT, point of subjective equality (PSE) and the just noticeable difference (JND) for each participant (figure 1). We have previously demonstrated that the PSE represents the most sensitive measure of temporal discrimination (Butler et al 2015); thus the PSE was of particular interest in comparing any variance in temporal discrimination during two consecutive menstrual cycles. 


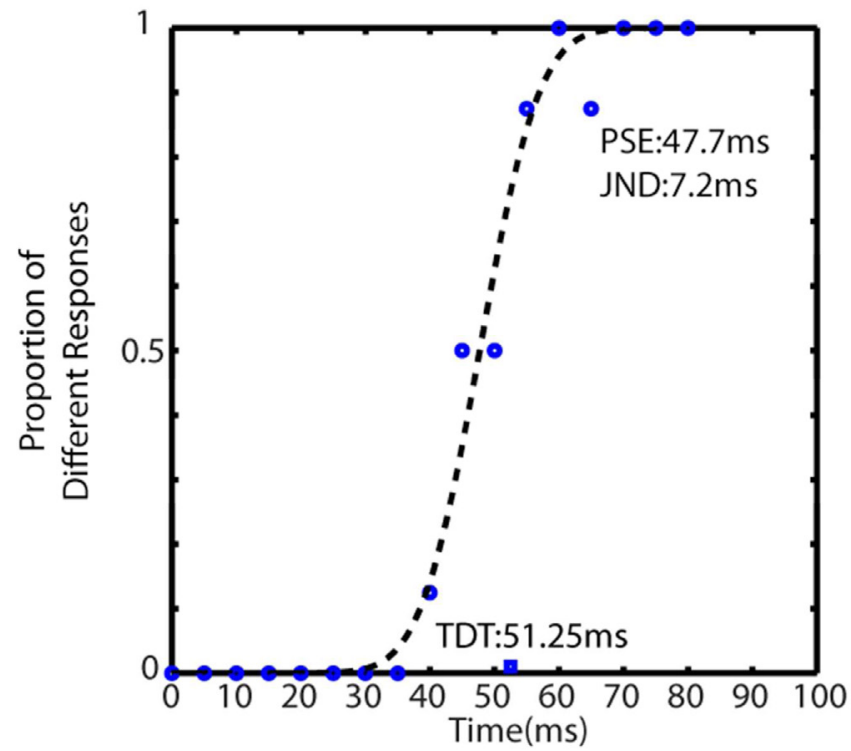

Figure 1. Data from one participant demonstrating the cumulative Gaussian psychometric fit. The blue circles show the proportion of perceived 'different' responses as a function of temporal asynchrony. The dashed curve represents the average cumulative Gaussian function. The blue square on the $X$-axis is the temporal discrimination threshold.

\section{Variance analysis}

A one-way repeated measure ANOVA was used to compare any variance in mean TDT Z-score and PSE across each menstrual quartile (quartile 1-4) and each week of testing (week 1-9). The independent variables used for this analysis were menstrual quartile and week of testing; the dependent variables were the TDT Z-score and PSE. All ANOVAs were carried out twice, with and without inclusion of the first week of testing. The first week of recording was discarded to allow for practice effect.

\section{Correlation analysis}

Correlation analysis was carried out to explore the relationship between TDT Z-score with menstrual quartile. The menstrual quartile was expressed as a dichotomous variable ( $1=$ quartile of interest and $2=$ other quartiles). The first quartile was the quartile of interest and it corresponded to the early-follicular phase. Quartiles two to four accounted for the other quartiles. A coefficient of determination was calculated to assess how much variance the two variables shared. Correlation tests were carried out twice, once with inclusion of week one of testing and once without. The first week of recording was discarded to allow for practice effect.

\section{Results}

Fourteen participants completed the study. The mean age of study participants was 19 years ( $\mathrm{SD} \pm 0.99$ years). Menstrual cycle length varied between participants (mean $32 \mathrm{~d}, \mathrm{SD} \pm 6.93$ d). The mean TDT Z-score was 0.78 (range: -1.19-3.14) and mean PSE was $40.13 \mathrm{~ms}$ (SD 14.42). The means and standard deviations are presented in table 1. 
Table 1. Temporal discrimination thresholds (TDT) (in milliseconds and Z-scores) and point of subjective equality (PSE) in 14 healthy control female participants recorded at weekly intervals over the eight weeks of two menstrual cycles. Quartile one results were obtained in the first quartile of the menstrual cycle.

\begin{tabular}{lcccc}
\hline & Quartile 1 & Quartile 2 & Quartile 3 & Quartile 4 \\
\hline Mean TDT (SD) (ms) & $43.7(15.5)$ & $41.8(15.5)$ & $43.9(13.6)$ & $45.7(13.2)$ \\
Mean TDT Z-score & 0.77 & 0.65 & 0.79 & 0.89 \\
Mean PSE (ms) & $40.4(13.2)$ & $42.6(14.9)$ & $43.0(14.5)$ & $42.2(17.4)$ \\
\hline
\end{tabular}

A one-way repeated measure ANOVA was conducted to compare TDT Z-scores and PSE between quartiles 1-4. The analysis revealed no significant effect for menstrual quartile on TDT Z-score (Wilks' Lambda $=0.95, F(3,13)=0.376, p=0.771$, multivariate partial eta squared $=0.049$ ). Mean TDT $Z$-score did not vary across menstrual cycle quartiles (figure 1 ).

A one-way repeated measures ANOVA was repeated to compare TDT Z-scores across each week of testing. There was no significant effect for week of testing on TDT Z-score (Wilks' Lambda $=0.36, F(7,13)=1.75, p=0.239$, multivariate partial eta squared $=0.636)$. For the PSE, the results demonstrated no significant effect for menstrual quartile on PSE (Wilks' Lambda $=0.83, F(3,13)=0.753, p=0.541$, multivariate partial eta squared $=0.158)$. There was no significant effect for week of testing on PSE (Wilks' Lambda $=0.25, F(7,13)=2.5$, $p=0.138$, multivariate partial eta squared $=0.748$ ).

The relationship between the TDT Z-score and menstrual quartile $(1=$ quartile of interest, $2=$ other quartile) was investigated using a point bisserial correlation. Preliminary analysis was performed to ensure no violation of the assumption of normality, linearity and homoscedasticity. There was a lack of correlation observed between the two variables. $\left(r_{\mathrm{pb}}=0.004\right.$, $n=112, p<0.968)$.

The dichotomous variable, menstrual quartile, demonstrated a lack of variability in mean TDT score. The coefficient of determination for the two variables (TDT Z-score and menstrual quartile) was low $\left(r^{2}=0.0000016\right)$, therefore demonstrating a lack of overlap between the two variables.

\section{Discussion}

In this study we have observed that temporal discrimination is unaffected by monthly fluctuations in sex-hormone levels. We have demonstrated no statistically significant variation in weekly temporal discrimination values during two consecutive menstrual cycles. Temporal discrimination was independent of menstrual quartile by correlation analysis and its mean did not vary between menstrual cycle quartiles. When comparing the temporal discrimination with of our quartile of interest (quartile one) with other quartiles, there was no statistically significant difference. As mentioned, our quartile of interest, is hormonally similar to menopause and is accompanied by reduced oestrogen and progesterone levels (Burger et al 1995). This observed stability of temporal discrimination values across menstrual quartiles suggests that temporal discrimination is unaffected by such hormonal fluctuations. Previous studies demonstrated that mean temporal discrimination in women is faster in women than men between 20 and 40 years of age but increases (worsens) progressively with age when compared with men (Butler et al 2015, Williams et al 2015b). Temporal discrimination in women deteriorates at almost three-times the rate of their age-matched male peers and thus, after 40 years of age, women have progressive worsening of their TDT compared to men. The lack of hormonal influence on temporal discrimination values observed in our study is important when considering the 


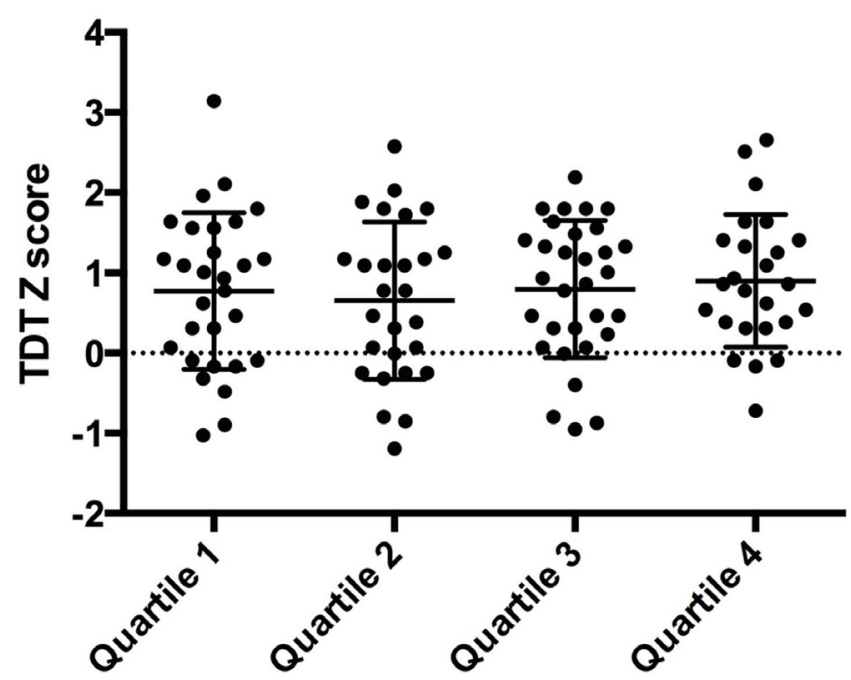

Figure 2. Temporal discrimination threshold (TDT) Z-scores in 14 female healthy controls during each of eight quartiles of two consecutive menstrual cycles. Quartile One is the quartile of interest. The filled circles from each quartile represents the TDT from this quartile.

pathogenesis of this sexually-dimorphic, age-related deterioration in temporal discrimination. The mean age of menopause is 51 years in women from industrialised countries (Gold 2011) and oestrogen levels remain within normal range during the perimenopausal transition (Rannevik et al 2008). Therefore, the female transition into hormonal senescence is 10 years after the sexually-dimorphic age-related decline observed in temporal discrimination (Williams et al 2015b). Therefore, we propose that this age-related deterioration in women's visual TDT scores is unlikely to be related to menopausal hormonal change. The findings from this study, that temporal discrimination is unaffected by cyclical-hormonal fluctuations, corroborates this hypothesis; which would be further supported by studies examining menopausal hormonal change on temporal discrimination. Our results lead us to consider an alternative explanation for this age-related sexual dimorphic deterioration in temporal discrimination. The TDT represents an individual's ability to detect environmental change. The midbrain covert attentional network plays a key role in the detection of salient stimuli in our environment (Fecteau and Munoz 2006, Redgrave et al 2010). We consider an abnormal TDT is due to a disorder of this network for covert attentional orienting caused by reduced gamma-aminobutyric acid (GABA) inhibition (Hutchinson et al 2014). A magnetic resonance spectroscopy study in healthy participants demonstrated a sexually-dimorphic, age-related decline in frontal GABA (Gao et al 2013). Above the age of 40 years women exhibited a more marked reduction in frontal GABA compared with men. This decline in frontal GABA mirrors the age-related deterioration in TDT scores observed in women. We suggest that the sexually-dimorphic differences observed in TDT above the age of 40 results from age- and sex-chromosome-related alterations in GABA levels and are not due to hormonal influences (Butler et al 2015).

\section{Summary and conclusion}

Our study, which demonstrated a lack of variation in temporal discrimination values during cyclical hormonal fluctuations, raises interesting questions concerning the age-related 
sexually-dimorphic decline observed in temporal discrimination. Our findings pave the way for future studies exploring potential pathomechanisms for this age-related deterioration

\section{Acknowledgments}

This research was supported by grants from Dystonia Ireland, the Irish Institute for Clinical Neuroscience, the Foundation for Dystonia Research (Belgium) and the Trinity Centre for BioEngineering.

\section{Financial disclosures (previous 12 months)}

Eavan McGovern, Emer O'Connor, Ines Beiser, Laura Williams, John Butler, Shruti Narasimham, Brendan Quinlivan, Rebecca Beck and Richard Reilly have no funding sources, financial disclosures or conflict of interests to declare.

Michael Hutchinson: serves as associate editor of the Multiple Sclerosis Journal, has received speaker's honoraria from Biogen-Idec, Bayer-Schering and Novartis and receives research grants from Dystonia Ireland, the Health Research Board of Ireland (CSA-2012-5), Foundation for Dystonia Research (Belgium) and the Irish Institute of Clinical Neuroscience.

Sean O'Riordan reports receiving a speaker's honorarium from Abbvie.

\section{Ethics statement}

Written informed consent was obtained from all participants. The study was approved by the Medical Research Ethics Committee at St Vincent's University Hospital.

\section{References}

Burger H G, Dudley E C, Hopper J L, Shelley J M, Green A, Smith A, Dennerstein L and Morse C 1995 The endocrinology of the menopausal transition: a cross-sectional study of a population-based sample J. Clin. Endocrinol. Metab. 80 3537-45

Butler J S et al 2015a Age-related sexual dimorphism in temporal discrimination and in adult-onset dystonia suggests GABAergic mechanisms Front. Neurol. 6258

Butler J S, Molloy A, Williams L, Kimmich O, Quinlivan B, O'Riordan S, Hutchinson M and Reilly R B 2015b Non-parametric bootstrapping method for measuring the temporal discrimination threshold for movement disorders J. Neural Eng. 12046026

Fecteau J H and Munoz D P 2006 Salience, relevance, and firing: a priority map for target selection Trends Cogn. Sci. $10382-90$

Gao F et al 2013 Edited magnetic resonance spectroscopy detects an age-related decline in brain GABA levels Neuroimage 78 75-82

Gold E B 2011 The timing of the age at which natural menopause occurs Obstet. Gynecol. Clin. North Am. 38 425-40

Hutchinson M et al 2013 The endophenotype and the phenotype: temporal discrimination and adultonset dystonia Mov. Disorders 28 1766-74

Hutchinson M et al 2014 Cervical dystonia: a disorder of the midbrain network for covert attentional orienting Front. Neurol. 5 1-10

Kimmich O, Bradley D, Whelan R, Mulrooney N, Reilly R B, Hutchinson S, O'Riordan S and Hutchinson M 2011 Sporadic adult onset primary torsion dystonia is a genetic disorder by the temporal discrimination test Brain $1342656-63$

McKinlay S M, Brambilla D J and Posner J G 1992 The normal menopause transition Maturitas 14 103-15 
Mihm M, Gangooly S and Muttukrishna S 2011 The normal menstrual cycle in women Animal Reprod. Sci. 124 229-36

Molloy A, Kimmich O, Williams L, Quinlivan B, Dabacan A, Fanning A, Butler J S, Riordan S O, Reilly R B and Hutchinson M 2014 Brief Reports A Headset Method for Measuring the Visual Temporal Discrimination Threshold in Cervical Dystonia pp 1-5

Rannevik G, Jeppsson S, Johnell O, Bjerre B, Laurell-Borulf Y and Svanberg L 2008 A longitudinal study of the perimenopausal transition: altered profiles of steroid and pituitary hormones, SHBG and bone mineral density Maturitas 61 67-77

Redgrave P, Coizet V, Comoli E, McHaffie J G, Leriche M, Vautrelle N, Hayes L M and Overton P 2010 Interactions between the midbrain superior colliculus and the basal ganglia Front. Neuroanat. $41-8$

Soares F A, Salomoni S E, Veneziano W H, de Carvalho J L A, de O Nascimento F A, Pires K F and da Rocha A F 2011 On the behavior of surface electromyographic variables during the menstrual cycle Physiol. Meas. 32 543-57

Speroff L, Glass R H and Kase N G 1999 Clinical Gynecologic Endocrinology and Infertility 6th edn (Baltimore, MD: Lippincott Williams and Wilkins)

Sundström Poromaa I and Gingnell M 2014 Menstrual cycle influence on cognitive function and emotion processing-from a reproductive perspective Front. Neurosci. 8380

Williams L J, Butler J S, Molloy A, McGovern E, Beiser I, Kimmich O, Quinlivan B, O'Riordan S, Hutchinson M and Reilly R B 2015 Young women do it better: sexual dimorphism in temporal discrimination Front. Neurol. 6 1-8

Ycaza Herrera A and Mather M 2015 Actions and interactions of estradiol and glucocorticoids in cognition and the brain: implications for aging women Neurosci. Biobehav. Rev. 55 36-52 